\title{
Real-time pressure-temperature reaction studies of biological systems using small-angle neutron scattering technique.
}

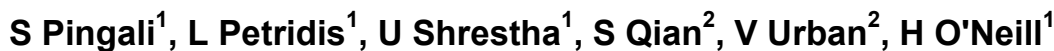 \\ ${ }^{1}$ Oak Ridge National Laboratory, Oak Ridge, TN, ${ }^{2}$ Oak Ridge National Laboratory \\ pingalis@ornl.gov
}

Scientific experiments that require the need to use extreme sample environments are ideally suited for neutron experiments due to the high penetration and non-destructive nature of biomass. The Bio-SANS instrument at Oak Ridge National Laboratory has developed a pressure cell that allows to heat the cell up to $300{ }^{\circ} \mathrm{C}$ as well as apply a maximum pressure of $1 \mathrm{kbar}$. Initial motivation for these capabilities were to track, in real-time, chemical reactions that span multiple hours and more recently expanded these capabilities to perform solution scattering of thermophilic and mesophilic proteins under pressure and/or temperature. Synergistically, the dynamic q-range for the detector system of the Bio-SANS instrument was improved from a factor of 20 to $~ 300$ by the installation of an additional detector array in the high scattering angle region. This talk will present the technical developments addressed to achieve these capabilities and two scientifically themed experiments will be presented. The first class of experiments will cover chemical reaction of plant biosystems and plant biopolymers. This will include dilute acid (acidic), sodium hydroxide (basic), co-solvents (acidic water/THF) as well as organic solvents (catalytic supercritical methanol) reactions required to breakdown plant biosystems. The presentation will cover new in-sights gained by these experiments that were not possible by the ex-situ based techniques. In fact, these measurements produced a paradigm shift in the thinking of biotechnologists pursuing biomass deconstruction strategies. The second set of experiments that is a relatively new capability, will cover solution scattering of thermophilic and mesophilic proteins. These measurements were carried out by varying the temperature, pressure and a combination of temperature/pressure of the protein solution system.

Acta Cryst. (2020). A76, a133 